\title{
Special issue on ant colony optimization
}

\author{
Karl F. Doerner • Daniel Merkle · Thomas Stützle
}

Received: 27 November 2008 / Accepted: 28 November 2008 / Published online: 17 December 2008 C) Springer Science + Business Media, LLC 2008

Ant Colony Optimization (ACO) is one of the most successful techniques in the wider field of swarm intelligence. ACO is inspired by the pheromone trail laying and following behavior of some ant species, a behavior that was shown to allow real ant colonies to find shortest paths between their colony and food sources. Taking many elements of the real ants behavior, foremostly their indirect communication through pheromone trails, the first ACO algorithms were proposed more than 15 years ago. Since then ACO has attracted a large number of researchers. In the first few years of ACO research, the focus was mainly on algorithmic advancements, trying to make ACO algorithms competitive with established metaheuristic techniques. Currently, the majority of the contributions concern successful applications of ACO algorithms to a variety of challenging problems; still, another active and productive research area in ACO is the theoretical study of the behavior of specific ACO algorithms.

The significant research efforts on ACO have established it as a mature metaheuristic that can lead to very effective algorithms for many difficult optimization problems. This special issue has the goal to collect papers on current, relevant work on ACO. We received 19 submissions on topics covering algorithmic developments, applications to continuous and combinatorial optimization problems, and theoretical studies. After a rigorous review process, three papers were selected for inclusion into this special issue. Among these, two

K.F. Doerner

Department of Business Administration, University of Vienna, Vienna, Austria

e-mail: karl.doerner@univie.ac.at

D. Merkle

Department of Mathematics and Computer Science, University of Southern Denmark, Odense, Denmark

e-mail: daniel@imada.sdu.dk

T. Stützle $(\bowtie)$

IRIDIA, CoDE, Université Libre de Bruxelles (ULB), Brussels, Belgium

e-mail: stuetzle@ulb.ac.be 
paper focus on theoretical contributions to the ACO field while the third reviews the currently available ACO algorithms for multi-objective optimization problems.

The first theoretical paper is entitled A Novel ACO Scheme for Optimization via Reinforcement and Initial Bias. The authors Vivek S. Borkar and Dibyajyoti Das introduce an ACO variant that is closer to real ants behavior than most state-of-the-art ACO algorithms. Their algorithm uses no external supervision and the pheromone update mechanism is based only on differential path length. With sophisticated mathematical methods, Borkar and Das show how a simple agent learning scheme helps in the convergence of the algorithm and why a large population size in the first iterations is important to guide the algorithm towards good solutions.

In the paper Analysis of Different MMAS ACO Algorithms on Unimodal Functions and Plateaus, Frank Neumann, Dirk Sudholt, and Carsten Witt present a rigorous runtime analysis for several variants of ACO algorithms. Their work addresses the question of how long it takes until the algorithm finds an optimal solution for a specific problem. They obtain lower and upper bounds on the runtime for the broad class of unimodal functions, they further tighten these bounds for specific unimodal functions, and they obtain new lower bounds for some plateau functions. What is particularly interesting in their work are the proof techniques they used to obtain the new lower bounds, techniques that can be extended to a broader class of optimization problems. Furthermore, the authors show that the right choice of a pheromone update mechanism can be the reason for the presence of a phase transition from polynomial to exponential runtime depending on the evaporation rate.

The third and last paper in this special issue, written by Daniel Angus and Clinton Woodward, reviews the current status of Multiple Objective Ant Colony Optimisation. The main contributions are the proposal of an extended taxonomy of ACO approaches to multiple objective optimization problems and a review of the currently available ACO algorithms for these problems in the light of this taxonomy. This taxonomy can help researchers to make a more informed choice about the available ACO algorithm components for tackling such problems.

\section{Acknowledgments}

The production of a special issue would not be possible without the help of a number of people. First, we want to thank all the authors who have submitted their work to this special issue. Second, we want to thank Marco Dorigo, the editor in chief of Swarm Intelligence, for the invitation to edit this special issue and his help in various issues arisen during the review process. Finally, we want to thank all the referees who contributed to this special issue with their timely and insightful reports.

Thomas Stützle acknowledges support from the F.R.S.-FNRS of the French Community of Belgium of which he is a Research Associate.

November 2008

Karl F. Doerner

Daniel Merkle

Thomas Stützle 